\title{
Residual stress measurement using a radial in-plane speckle interferometer and laser annealing: preliminary results
}

\author{
M. R. Viotti ${ }^{a}$, R. Sutério ${ }^{b}$, A. Albertazzi Jr. ${ }^{b}$, G. H. Kaufmann ${ }^{a, c_{*}}$ \\ ${ }^{a}$ Instituto de Física Rosario (CONICET-UNR), Bvd. 27 de Febrero 210 bis, \\ S2000EZP Rosario, Argentina \\ ${ }^{b}$ Laboratório de Metrologia e Automação, Universidade Federal de Santa Catarina, \\ CEP 88040-970, Florianópolis, SC, Brazil \\ ${ }^{c}$ Departamento de Física, Facultad de Ciencias Exactas, Ingeniería y Agrimensura, \\ Universidad Nacional de Rosario, S2000BTP Rosario, Argentina
}

\begin{abstract}
This paper presents a combined system based on digital speckle pattern interferometry and laser annealing to determine residual stresses in ductile materials. The system allows the measurement of the radial in-plane displacement field generated when a specimen subjected to residual stresses is locally heated. This novel approach for measuring residual stresses has the advantage of being nondestructive and can be easily applied to in situ tests. The description of the combined system is followed by the presentation of preliminary results which illustrate the ability of both techniques to identify residual stress fields and also to determine their principal directions.
\end{abstract}

Keywords: digital speckle pattern interferometry; residual stresses; nondestructive evaluation; laser annealing; radial interferometer.

\section{INTRODUCTION}

Residual stresses are present in many structures and mechanical components. ${ }^{1}$ They may be produced by fabrication operations such as casting, rolling, welding, heat-treating or forging, or may occur during the life of structures. These stresses exist without the action of external forces and they combine with service stresses. In consequence, this resulting combination affects the mechanical behavior of materials and may be the reason of the failure. Moreover, compressive residual stresses are intentionally introduced in some fabrication processes to increase fatigue resistance and also to improve other material properties.

In many applications, it is important to know the magnitude and direction of residual stresses. However, it is difficult to foresee them with accuracy because the history of the testing specimen from the fabrication of the raw material up to the component in service should be known. Therefore, the most adequate form to determine the level of residual stresses is the use of experimental methods.

One of the most widely used experimental methods is the hole-drilling technique. ${ }^{2}$ This technique involves monitoring the strains produced when a small hole is drilled into a stressed material. The strains released by hole drilling are usually measured with strain-gauge rosettes but their utilization has some drawbacks: (a) the necessity of a plane and smooth surface to bond the rosettes, (b) the preparation of the material surface, (c) the high cost and time associated with the installation of rosettes, and (d) the requirement that the hole be drilled exactly in the center of the rosette in order to avoid sizeable errors.

Due to these difficulties, non contacting optical techniques have been applied over the last two decades to measure the displacement field around the hole. These techniques generate fringe patterns from which the local displacements in the neighborhood of the hole can be calculated. ${ }^{3}$ The use of moiré interferometry for residual stresses determination was investigated by McDonach et al. ${ }^{4}$ However, bonding a grating can also be time-consuming. The feasibility of using holographic interferometry to measure both in-plane and out-of-plane displacement caused by hole drilling was reported

* Send correspondence to G.H. Kaufmann

E-mail: guille@ifir.edu.ar. Fax: 54341 482-1772 
by Antonov, ${ }^{5}$ Nelson and Mc Cricked, ${ }^{6}$ Makino and Nelson. ${ }^{7}$ More recently, Zhang ${ }^{8}$ investigated the practicability of the combination between digital speckle pattern interferometry (DSPI) and hole drilling. As it is well known, DSPI is based on the correlation of speckle interferograms generated by a laser source. The attractiveness of this technique to the optical metrology community arises not only from its non-contacting nature but also from the relative speed of the inspection procedure, mainly due to the use of video detection and digital image processing.

In all the previous papers, fringe analysis was carried out by laborious manual reduction and the value of the residual stress was determined by means of fringe counting methods using a very small number of measurements. A hole drilling and DSPI combined system with automated data analysis was recently presented by Díaz et al. ${ }^{9}$ The inplane displacement field generated by the introduction of the hole was determined from the calculation of the optical phase distribution using a phase shifting method and a robust iterative phase unwrapping algorithm. This combined system enables the measurement of whole-field displacements in the neighborhood of the hole, allowing the application of a least-squares fitting method to determine the residual stress value.

For the general case in which the direction of the residual stresses is not known, a speckle interferometer with two dual-beam illumination beams must be used to enable the separate measurement of both in-plane displacement components. Even though some DSPI systems based on polarization discrimination methods were developed for this purpose, ${ }^{10,11}$ this approach has two drawbacks: (a) the test surface can appreciably depolarize the two orthogonalpolarized dual-beam illumination sets causing cross interference between them; (b) the optical setup becomes more bulky and complex. A step forward to solve these problems was recently given by Albertazzi et al. ${ }^{12}$ with the development of a novel double illumination DSPI system to measure radial in-plane displacements. This radial interferometer was combined with the hole drilling technique to obtain a portable device which measures residual stresses outside of the optical bench. This system allows the measurement of the principal residual stresses and their direction from the analysis of only one correlation fringe pattern.

Although the potential of hole drilling to measure residual stresses has been firmly established, and its portability, accuracy and range of measurement make it quite suitable for analyzing mechanical components located outside of the laboratory, this technique is a semi-destructive one. For this reason, other nondestructive techniques for relieving residual stresses have been recently tested. Among them, perhaps local annealing is the one that has more possibilities of success. The technique is based on the idea that the level of residual stresses will decrease when a stressed material is heated. The degree of relief stress will depend on the time and temperature of the heat treatment, and also on the stressstrain characteristics of the material that is heated.

The application of local heating for releasing the residual stress combined with DSPI for measuring the in-plane displacement component was recently presented by Perchersky et al. ${ }^{13,14}$ Using preloaded test specimens under uniaxial tension and a simple theoretical model to explain the annealing process, through a series of experiments these authors demonstrated the validity of this approach for releasing residual stresses. As the loading direction was known, a standard dual-beam interferometer with a sensitivity along that direction was used in these works. However, fringe patterns obtained from local annealing experiments were analyzed by manual reduction, procedure which is timeconsuming and also has low accuracy.

This paper presents the description of a combined system of DSPI and laser annealing that uses a radial in-plane interferometer similar to the one presented in Ref. 12 and a carbon dioxide laser as a heating source. By means of this combined system, the stresses existing in the specimen under investigation are released and the subsequent radial inplane displacement field generated by the heating process is measured using the DSPI technique. The description of the radial in-plane speckle interferometer and the laser annealing combined system is followed by the presentation of some preliminary results which illustrates the advantages of this nondestructive residual stress measurement approach.

\section{PRINCIPLES OF THE RADIAL IN-PLANE INTERFEROMETER}

Figure 1 shows the basic setup to obtain radial in-plane sensitivity. The main element of the interferometer is a conical mirror, which is placed near the specimen surface. The figure shows a cross-section of the conical mirror containing the mirror axis, which displays two particularly chosen light rays from a collimated illumination source. Each light ray is reflected by the conical mirror surface towards a point $\mathrm{P}$ over the specimen surface, reaching this point symmetrically. The illumination directions are indicated by the unitary vectors $\mathbf{n}_{\mathbf{A}}$ and $\mathbf{n}_{\mathbf{B}}$ and they have the same angle with respect to the axis of the conical mirror. The sensitivity direction is given by the vector $\mathbf{k}$ obtained from the subtraction of the two unitary vectors. Therefore, in-plane sensitivity is reached in point P. In the same cross-section and for any other point 
given on the specimen surface, it can be verified that there is only one couple of light rays that merge at that point. The incidence angle is always the same and symmetric respect to the mirror axis. If the surface normal is coincident with the axis of the conical mirror, the unitary vectors $\mathbf{n}_{\mathbf{A}}$ and $\mathbf{n}_{\mathbf{B}}$ will have the same angle $\beta$ with respect to the surface normal. Therefore, for each point at the surface of the specimen for this cross-section, the sensitivity vector $\mathbf{k}$ will be parallel to the specimen surface and a radial in-plane sensitivity will be obtained.

The above description can be extended to other cross-sections of the conical mirror. If the central point is kept out from this analysis, it can be demonstrated that each point of the specimen surface is illuminated by only one pair of light rays. Both rays are coplanar with the mirror axis and symmetrically oriented to it. Therefore, fully $360^{\circ}$ radial sensitivity is obtained for a circular region over the specimen.

A practical configuration of the radial in-plane interferometer is shown in Fig. 2. The laser light is expanded and collimated by two convergent lenses. The collimated beam is reflected towards the conical mirror by means of a mirror that forms a $45^{\circ}$ angle with the axis of the conical component. The central hole located at this mirror has two main functions: (a) to avoid that the laser light reaches directly the sample surface and to prevent triple illumination, and (b) to provide a viewing window for the CCD camera.

The intensity of the light is not constant over the whole illuminated area over the specimen surface. It is particularly higher in the central point because this singular point receives light contribution from all cross-sections. Therefore, a very bright spot is visible in the central part of the circular measurement region and it would reduce fringe contrast. To reduce this effect, the conical mirror is designed to have the split configuration shown in Fig. 1. It is formed by two parts with a small gap between them. The distance of this gap is adopted in such a way that the light rays reflected to the center are blocked. Thus, a small circular shadow is created in the center of the illuminated area and fringe blurring is avoided.

As it can be seen from Fig. 1, the two rays of the double illumination for each point over the specimen are from the reflection of the upper and lower parts of the conical mirror. A piezoelectric actuator (PZT) was used to join the upper part of the conical mirror. The lower part of the conical mirror is fixed while the upper part is mobile. Therefore, the PZT moves the upper part along its axial direction and the gap between both parts is increased. In consequence, a small optical path change between both rays light that intersect on each point is produced. The PZT device allows the introduction of a phase shift to calculate the optical phase distribution by means of the Carré algorithm. ${ }^{15}$

\section{EXPERIMENTAL SETUP AND TESTING PROCEDURE}

According to the diagram shown in Fig. 2, the radial in-plane interferometer was implemented as follows. A $50 \mathrm{~mW}$ diode laser with a wavelength of $780 \mathrm{~nm}$ was used as a light source. The directions of illumination $\mathbf{n}_{\mathbf{A}}$ and $\mathbf{n}_{\mathbf{B}}$ are displayed in Fig. 1. The angle $\beta$ between them and the normal to the specimen surface was chosen as $60^{\circ}$. To introduce the phase shifts, three piezoelectric transducers were joined to the upper part of the conical mirror. The test specimen was monitored lively by a CCD camera. It provided a field of view that included the illuminated area of $10 \mathrm{~mm}$ in diameter over the specimen.

In order to produce the local annealing, a carbon dioxide laser with a power of $10 \mathrm{~W}$ and a wavelength of $10.6 \mu \mathrm{m}$ was used. The heated surface was a circular spot with a diameter of $1.6 \mathrm{~mm}$. To obtain this heat spot, the $\mathrm{CO}_{2}$ laser beam was guided and focused using a mirror and a $\mathrm{ZnSe}$ lens with short focal length.

The heating process must be realized carefully since nonlinear response of the material could be introduced. If the material is heated too much or too fast, the local thermal expansion will create plastic compressive strains. ${ }^{13}$ Therefore, there would be two types of strains: one due to the residual stress relaxation and the other due to the induced plastic compressive deformation which would introduce significant errors in the measurements of the residual stresses. They can be avoided by using an appropriate heating cycle. Hence, the following heating cycle was chosen: the $\mathrm{CO}_{2}$ laser heated the specimen at approximately half of its power for a period of 20 seconds. This cycle created a power density in the heating area of $260 \mathrm{~W} / \mathrm{cm}^{2}$ and increased the local temperature of the specimen to approximately $350{ }^{\circ} \mathrm{C}$. This temperature was computed using a heat propagation calculation and assuming a specific heat constant of $900 \mathrm{~J} / \mathrm{kg}{ }^{\circ} \mathrm{K}$ for the aluminum specimen. As the measured residual stress depends on the temperature rise due to the laser heating, an adequate temperature sensor device has to be used to obtain quantitative values of the residual stresses.

To perform the measurements, the following experimental procedure was applied. First, the radial in-plane interferometer was positioned over the specimen to be tested by means of three adjustable legs. These legs and the 
specimen were clamped on the surface of an optical bench in order to keep negligible the relative displacement between the interferometer and the specimen. Then, a set of 4 phase-shifted speckle interferograms was acquired and the reference phase distribution was computed and stored. Afterwards, the specimen was locally heated and another set of 4 phase-shifted speckle interferograms was acquired. The wrapped phase distribution was calculated from the difference between the initial and final phase distributions. The difference of phase method was used because it gives a significant improvement in the quality of the reconstructed phase maps when comparing with the phase of differences method. ${ }^{15}$ This approach also allows the use of a local smoothing operation to improve the signal-to-noise ratio of pixels having very low intensity modulation.

The continuous phase distribution was obtained using a block least-squares phase unwrapping algorithm proposed by Strand. ${ }^{16}$ Once the phase unwrapping was made, the radial in-plane displacement field $u_{r}(r, \theta)$ can be calculated from the optical phase distribution $\phi(r, \theta)$ by ${ }^{17}$

$$
u_{r}(r, \theta)=\frac{\phi(r, \theta) \lambda}{4 \pi \sin \beta}
$$

where $\lambda$ is the wavelength of the laser and $\beta$ is the angle between the illumination directions and the normal direction of the specimen surface.

When the hole drilling technique is used, the principal residual stresses $\sigma_{1}$ and $\sigma_{2}$ and the principal direction angle $\alpha$ can be calculated from the radial in-plane displacement $u_{r}(r, \theta)$ using an analytical solution developed by Kirsch. ${ }^{12}$ This model is based on the elastic solution for an infinite plate subjected to an uniform stress field in which a cylindrical hole is drilled through the plate thickness. In this case, the relationship between the radial in-plane displacement and the residual stresses can be written as

$$
u_{r}(r, \theta)=A(r)\left(\sigma_{1}+\sigma_{2}\right)+B(r)\left(\sigma_{1}-\sigma_{2}\right) \cos (2 \theta-2 \alpha)
$$

where $A(r)$ and $B(r)$ are functions of the hole radius and the material elastic constants. Taking into account that the radius of the drilled hole can be experimentally evaluated, the residual stresses can be determined from the measurement of the radial displacement $u_{r}$ along a circle of radius $\rho$ concentric with the hole and the axis of the conical mirror. As thousands of these measurements are usually available, the magnitude and direction of the residual stresses can be determined using a harmonic method based on Fourier analysis, approach which allows to minimize random errors. $^{18}$

When the residual stresses are relaxed by local heating, unfortunately no analytical solution exists for obtaining the residual stresses from the radial displacement field. However, a numerical solution to this problem can be reached by means of a method such as finite elements if the spot size, heating cycle, heat flux, pulse width, mechanical and thermal constants of the material are known. As the main objective of this work is to present the DSPI-local heating combined system and to demonstrate its feasibility to identify residual stresses regions, the quantitative determination of the residual stress value from the displacement field measured with the radial interferometer will be reported in a next paper.

\section{EXPERIMENTAL RESULTS}

We selected a simple aluminum plate with a rectangular cross-section as specimen. The plate was plastically deformed by a punctual compression load to generate residual stresses. Therefore, after unloading, permanent stress remained in the region of the specimen around the point of application of the load. In regions away from this point, the specimen did not present induced residual stresses.

We analyzed the influence of the thermal expansion produced by the local annealing on the measurements through preliminary experiences and we concluded that the number of fringes did not vary with time for intervals longer than 10 $\mathrm{s}$. As consequence, a time interval of $10 \mathrm{~s}$ from the end of the heating cycle was adopted for acquiring the second set of phase-shifted interferograms.

A typical wrapped phase map corresponding to a region without residual stresses obtained $10 \mathrm{~s}$ after the heating cycle finished is shown in Fig. 3(a). As this image presents only one fringe which covers the whole illuminated area, it can be seen that in this case the local heating process produces a minimal perturbation. On the contrary, Fig 3(b) shows 
the wrapped phase map that resulted from the local annealing on a region with residual stresses. This phase map was also obtained $10 \mathrm{~s}$ after the local annealing cycle finished.

By comparing Figs. 3(a) and 3(b), it can be observed that the combined system proposed in the present paper simply allows to identify regions with and without residual stresses. It is also very important to note that due to the radial symmetry of the in-plane interferometer, as it is shown in Fig. 3(b), it is not a difficult task to determine the principal direction of the residual stresses from the wrapped phase maps.

\section{CONCLUSIONS}

The DSPI system combined with local laser annealing presented in this paper enables in situ measurement of residual stresses. The system uses a $\mathrm{CO}_{2}$ laser as a heating source to produce stress relaxation and a radial in-plane interferometer to measure the displacement field generated around the heating spot. Compared to the hole-drilling technique, local annealing is a very interesting method to release residual stresses because it is non-contact, nondestructive and also cleaner than the drilling process since no metal chips are produced.

According to the preceding analysis, it can be concluded that the combined system allows a simple identification of regions with residual stresses from the free ones. If an adequate heating cycle is applied, this system introduces a very low level of additional residual stresses. Also, due to the radial symmetry of the in-plane interferometer, the principal directions of the residual stress field can be easily determined from the wrapped phase maps. Therefore, it is not necessary to use a DSPI system with a special design for measuring released displacements along two orthogonal directions.

In spite of the encouraging results, it can be seen that the utilization of local annealing in the present work is limited to qualitative evaluation of residual stress fields. Additional investigations are necessary for quantitative evaluation. They are taking place and they involve a numerical solution to this problem with a finite element model where the spot size, heating cycle, heat flux, pulse width, mechanical and thermal constants of the material are known. These investigations will be the object of a future work.

\section{ACKNOWLEDGMENTS}

M. R. Viotti would like to thank the financial support of the Multipurpose Optical Network, funded by The International Centre of Theoretical Physics, Trieste, Italy, for visiting the Universidade Federal de Santa Catarina.

\section{REFERENCES}

1. M. R. James and J. Lu, "Introduction," in Handbook of Measurement of Residual Stresses, J. Lu, Ed., pp. 1-4, The Fairmont Press, Lilburn, GA (1996).

2. ASTM E387-92, "Standard test method for determining residual stresses by the hole-drilling strain-gage method," in Annual Book of ASTM Standards, Section 3, Vol. 03.01. Am. Soc. Test. Mat., 747-753 (1992).

3. F. M. Furgiuele, L. Pagnotta, and A. Poggialini, "Measuring residual stresses by hole drilling and coherent optics techniques: a numerical calibration," J. Eng. Mat. Tech. 113, 41-50 (1991).

4. A. McDonach, J. McKelvie, P. M. MacKenzie, and C. A. Walker, "Improved moiré interferometry and applications in fracture mechanics, residual stresses and damage composites," Exp. Tech. 7, 20-24 (1993).

5. A. A. Antonov, "Inspecting the level of residual stresses in welded joints by laser interferometry," Weld. Prod. 30, 29-31 (1983).

6. D. V. Nelson and T. T. Mc Crickerd, "Residual stress determination through combined use of holographic interferometry and blind hole drilling," Exp. Mech. 26, 371-378 (1986).

7. A. Makino and D. Nelson, "Residual-stress determination by single-axis holographic interferometry and hole drilling. Part I: theory," Exp. Mech. 34, 66-78 (1994).

8. J. Zhang, "Two-dimensional in-plane electronic speckle pattern interferometer and its application to residual stress determination," Opt. Eng. 37, 2402-2409 (1998).

9. F. V. Díaz, G. H. Kaufmann, and G. E. Galizzi, "Determination of residual stresses using hole drilling and digital speckle pattern interferometry with automated data analysis," Opt. Lasers Eng. 33, 39-48 (2000).

10. A. J. Moore and J. R. Tyrer, "An electronic speckle pattern interferometer for complete in-plane measurement," Meas. Sci. Tech. 1, 1024-1030 (1990). 
11. A. J. Moore and J. R. Tyrer, "Two-dimensional strain measurement with ESPI," Opt. Lasers Eng. 24, 381-402 (1996).

12. A. Albertazzi Jr., M. R. Borges, and C. Kanda, "A radial in-plane interferometer for residual stresses measurement using ESPI," Proc. SEM IX Int. Congress on Exp. Mech., pp. 108-111, Society for Experimental Mechanics, Bethel, CT (2000).

13. M. J. Pechersky, R. F. Miller, and C. S. Vikram, "Residual stress measurements with laser speckle correlation interferometry and local heat treating," Opt. Eng. 34, 2964-2971 (1995).

14. C. S. Vikram, M. J. Pechersky, C. Feng and, D. Engelhaupt, "Residual stress analysis by local laser heating and speckle correlation interferometry," Exp. Tech. 20, 27-30 (1996).

15. J. M. Huntley, "Automated analysis of speckle interferograms," in Digital Speckle Pattern Interferometry and Related Techniques, P. K. Rastogi, Ed., pp. 59-139, Wiley, Chichester (2001).

16. J. Strand, T. Taxt, and A. K. Jain, "Two-dimensional phase unwrapping using a block least-squares method," IEEE Trans. Image Process. 8, 375-386 (1999).

17. P. K. Rastogi, "Measurement of static surface displacements, derivatives of displacements and three-dimensional surface shapes. Examples of applications to non-destructive testing," in Digital Speckle Pattern Interferometry and Related Techniques, P. K. Rastogi, Ed., pp. 142-224, Wiley, Chichester (2001).

18. M. R. Rodacosky, "Residual stresses measurement with ESPI," (in Portuguese), Dr. Eng. Dissertation, Federal University of Santa Catarina", Brazil (1997).

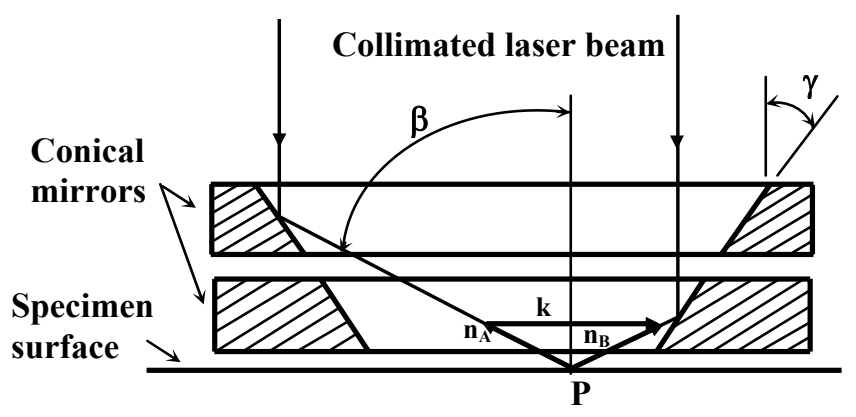

Figure 1. Cross-section of the upper and lower parts of the conical mirror to show the radial in-plane sensitivity of the interferometer.

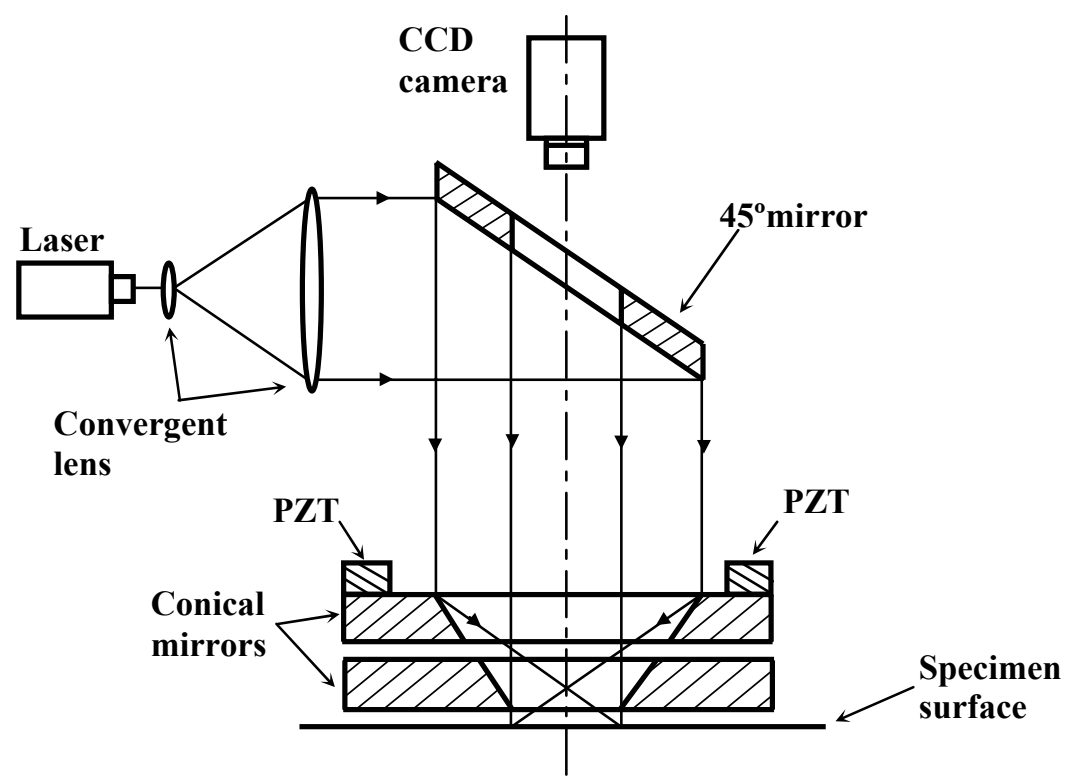

Figure 2. Optical arrangement of the radial in-plane interferometer. 


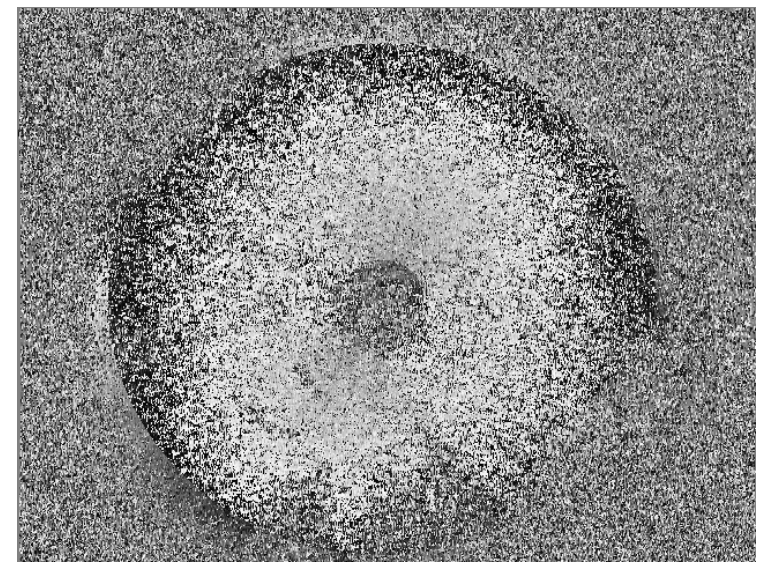

(a)

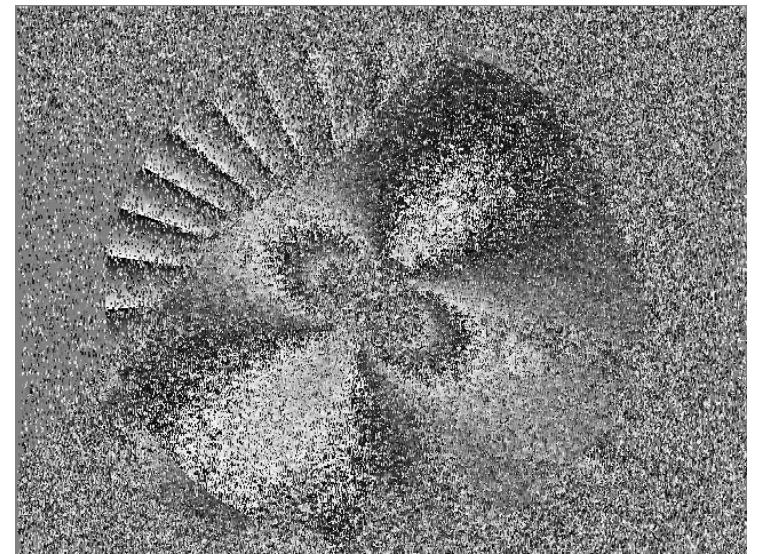

(b)

Figure 3. Wrapped phase map obtained $10 \mathrm{~s}$ after the heating cycle was finished: (a) in a region without residual stresses; (b) in a region with residual stresses. 